\title{
JNO special issue: an update on pediatric neuro-oncology
}

\author{
L. B. Kilburn ${ }^{1,2} \cdot$ Roger J. Packer ${ }^{1,3}$ (])
}

Received: 28 May 2020 / Accepted: 13 June 2020 / Published online: 26 August 2020

(c) Springer Science+Business Media, LLC, part of Springer Nature 2020

In spite of tremendous advances in our Queryunderstanding of the biology of many pediatric brain tumors, they remain the leading cause of death due to cancer in children [1]. Significant progress has been made in better stratifying patients based on both clinical and molecular tumor characteristic resulting in improvements in the long term survival, quality of life or late effects in some children with tumors such as medulloblastoma or low grade glioma. However there remain many pediatric brain tumor types for which little or no therapeutic progress has been made over many decades. New therapeutic approaches remain greatly needed.

Over the last two decades the critical role of epigenetics in cancer biology has evolved significantly. In parallel our understanding of the biology of many pediatric brain tumors and the central role of alterations in their epigenetic regulation has become an important area of discovery. Hopefully over the next decade the biologic advances, which are detailed throughout this issue, in understanding the role of epigenetics will further characterize pediatric brain tumors, open new therapeutic avenues for treatment and lead to true breakthroughs and cures for children [2].

Research toward understanding the balance between transcriptional activation and repression through chromatin modifications and the role of epigenetic regulation in cancer development, maintenance and resistance to therapy continues to evolve. Starting with the early recognition that cancers had abnormal gain and loss of methylation, through identification of gene mutations in promoter regions or components of histones or chromatin remodeling complexes across cancer types, to more recent tumor specific research into

Roger J. Packer

rpacker@cnmc.org

1 Brain Tumor Institute, Children's National Hospital, Washington, DC, USA

2 Center for Cancer and Blood Disorders, Children's National Hospital, Washington, DC, USA

3 Center for Neuroscience and Behavioral Medicine, Children's National Hospital, Washington, DC, USA the implications of these changes on tumor phenotype and microenvironment, our increased understanding of the role of epigenetics in cancer addresses unanswered questions but also lead to new areas of research.

In contrast to many adult tumors, which demonstrate distinct tumor mutations and higher mutational burden, the paucity of recurrent mutations in pediatric cancers, have often been highlighted [3]. Embryonal tumors like medulloblastoma, atypical teratoid rhabdoid tumor had long been recognized as biologically distinct from brain tumors which occur more commonly in adults and tumor clustering and categorization by genome-wide DNA methylation and gene expression analysis has further substantiated these differences. More recent discoveries of mutations in histone genes in pediatric glioma have also highlighted their biologically distinct nature and sparked further research in epigenetic regulation across the spectrum of pediatric brain tumors. Specifically identification of mutations in histone genes in a majority of pediatric high grade gliomas and BRAF pathway alterations in pediatric pilocytic astrocytomas highlight how biologically unique most pediatric tumors are from even histologically similar adult brain tumors [4, 5]. Many of the recent discoveries in pediatric brain tumor biology have centered on alterations in epigenetic modifiers or heritable changes in the epigenome, including changes in DNA methylation, chromatin remodeling, microRNAs and long coding RNAs. Alterations in epigenetic modifiers have been identified in a variety of pediatric brain tumors over the last decades including high grade glioma, atypical teratoid rhabdoid tumors (ATRT), ependymoma and medulloblastoma and research in this area has improved our understanding of the biology of these tumors. Unfortunately, however, these advances have not yet effectively translated to novel effective therapies. As scientists continue to unravel the complex implications of these epigenetic changes on the development and maintenance of pediatric brain tumors, the next challenge will be harnessing that knowledge toward rationale therapeutic approaches.

Identification of unique mutations in pediatric brain tumors have in some cases lead to new diagnostic entities, 
and in others to define subgroups within previously defined tumor types, as well as to point toward potential for novel targeted therapies. The 2016 update to the WHO diagnostic criteria incorporated molecular classifications including restructuring of diffuse glioma with incorporation of genetically defined entities, with diffuse midline glioma, H3K27M mutant (WHO grade IV) now defined as a distinct entity in addition to IDH-mutant glioma, both entities which lead to important epigenetic changes within the tumor. Further updates for the WHO diagnostic criteria included restructuring of both medulloblastoma and embryonal tumors to incorporate molecular entities including the classification of WNT and SHH mutant medulloblastoma and addition of other molecularly defined entities including embryonal tumor with multilayered rosettes, C19MC-altered and removal of the term primitive neuroectodermal tumors [6]. Several of these more recently recognized mutations lead to altered tumor epigenetics.

One of the earliest recognized mutations in pediatric brain tumors with implications in epigenetics was in ATRT. Mutations in SMARCB1 (SWI/SNF-related matrix-associated actin-dependent regulator of chromatin subfamily B member 1/hSNF5 /INI1) were recognized early in a majority of ATRTs, however understanding implications on the biology of these tumors and translation into novel therapies took more time. In ATRT these recurrent mutations in SMARCB1 were identified in a majority of tumors as well as other rhabdoid tumors almost two decades ago [7]. However an understanding of the role of SMARCB1/INI1 and its complex role in the SWI/SNF complex and thus contribution to cancer has evolved over the last decade [8]. The interplay between the epigenetic regulators Polycomb and SWI/SNF complexes is important in normal development, and loss of SMARCB1/INI1 as seen in rhabdoid and other tumors leads to elevated expression of the polycomb gene enhancer of zeste holomlog 2 (EZH2). EZH2 is the enzymatic component of the polycomb repressive complex 2 (PRC2) and is responsible for the $\mathrm{H} 3 \mathrm{~K} 27$ trimethylation activity of the complex [9]. In tumors with INI1 loss, there is resultant H3K27 trimethylation and repression of target genes, which leads to activation of stem cell associated programs. Further, inhibition of INI1 in tumor cell lines results in tumor growth inhibition [9]. This evolution in understanding of the role of epigenetics uncovered potential therapeutic targets in these often resistant tumors. Tazemetostat is a selective inhibitor of the histone methytransferase EZH2. When tested in the pediatric preclinical testing program, tazemetostat demonstrated efficacy in rhabdoid tumor xenograft lines and was noted to demonstrate significant reduction of H3K27me3 levels in the majority of tumors [10]. Tazemetostat has subsequently received FDA approval for the treatment of advanced epitheloid sarcoma, another rare tumor with INI1 loss. Early phase clinical trials of tazemetostat in children with ATRT and other EZH2, SMARCA1 and SMARCA4 deficient tumors are ongoing with early reports suggesting potential efficacy(NCT02601937, NCT03213665) [11]. Incorporating these types of epigenetic modifiers into more traditional chemotherapeutic approaches will be important next steps.

Similarly, further investigation into the relatively genetically quiet group three and four medulloblastomas has also identified alterations in demethylases, acteyltransferases, and nucleosome remodelers as well as aberrant $\mathrm{H} 3 \mathrm{~K} 4$ and HK27M methylation [12]. There is little doubt that epigenetics will play a crucial role in the understanding and therapeutic approaches to other embryonal tumors.

Similar to ATRT, alterations in trimethylation have been highlighted in diffuse midline gliomas. The identification of recurrent histone mutations in a majority of midline gliomas, transformed the thinking of how to approach pediatric gliomas and solidified their recognition as biologically quite distinct from adult gliomas. As noted above, alterations in histone genes are recognized as the most common mutation in children with diffuse midline gliomas. This recently defined diagnostic entity, diffuse midline glioma, K27M mutant includes both the historically clinically diagnosed diffuse intrinsic pontine glioma (DIPG) as well as thalamic high grade gliomas. It is now recognized that $\mathrm{K} 27 \mathrm{M}$ mutant tumors in the thalamus harbor similar outcome to those in children with DIPG. Mutations in the histone genes H3F3A were initially identified in children with DIPG and glioblastoma less than a decade ago as well as mutations in ATRX ( $\alpha$-thalassaemia/mental retardation syndrome $\mathrm{X}$-linked) and DAXX (death-domain associated protein) [13, 14]. This discovery defined pediatric glioma as biologically unique from adult tumors and pointed to defects in chromatin architecture as potentially underlying pediatric gliomagenesis [14]. The global changes in methylation that occur in these tumors are profound and may contribute to their resistance to chemotherapy and radiation therapy. In a chemical screen of patient derived DIPG cell cultures histone deacetylase inhibitors (HDACi) were identified as potentially effective therapies. Subsequent in vitro and in vivo studies of panobinostat supported the potential efficacy of this agent which is now being evaluated in a phase I trial in children with DIPG [15]. NCT02717455 as well as by convection enhanced delivery in a nanoparticle formulation (NCT03566199). Preclinical evaluations of other epigenetic modifiers, including histone demethylase inhibitors, BET inhibitors, EZH2 inhibitors have also shown promise in DIPG and HGG models and are promising alternative approaches to therapy for this difficult disease.

Interestingly, although ependymomas lack the histone mutations seen in children with diffuse midline glioma (DMG), a subgroup of these tumors, the posterior fossa A (PFA) group, similarly demonstrate loss of the H3K27 
methyl mark [16]. Although they lack recurrent single nucleotide mutations or copy number variations, PFA ependymoma tumors were initially recognized to have more methylated $\mathrm{CpG}$ sites and more genes that are transcriptionally silenced compared to the those in PFB tumors [17]. It subsequently was identified that there was also global H3K27 trimethylation reduction in the PFA tumors similar to that seen in H3K27M mutant gliomas. This global reduction in H3K27Mme3 was inversely correlated with the CpG hypermethylation, and despite these changes the tumors showed overall hypomethylation in the rest of the genome in a similar patter to that seen in H3K27M mutant gliomas [18]. Subsequently the protein EZHIP was shown to inhibit PRC2 activity in ependymoma through a H3K27M like mechanism $[1,19]$ How these similarities can be leveraged from a therapeutic perspective remains to be defined, but children with PFA tumors also have a poor prognosis with frequent tumor recurrence and thus novel therapies which target these epigenetic alterations should be explored similarly to in children with DMG. A clinical trial that targets this epigenetic alteration using one of the earliest epigenetic chemotherapeutic agents, 5'-azacytidine, in combination with carboplatin in children with recurrent disease is currently underway (NCT03206021).

As research continues to investigate the role of epigenetics in other pediatric brain tumor subtypes, additional targets are likely to be identified. While significant progress has been made in identifying mutations in epigenetic modifiers or identifying evidence of altered epigenetic machinery in tumors, there remain many unanswered questions regarding how to best target these alterations therapeutically. While preclinical evaluations of therapies targeting these epigenetic pathways have shown some promise, translation of these approaches into the clinic remains in its infancy. Given the complexity of epigenetic modifications on gene expression and repression there remains significant research to be done. Even with new approaches, it is likely that multimodal therapy will be needed to adequately target these malignant tumors and how to best incorporate epigeneticbased therapies into standard approaches including potential impact of sequencing and combinatorial targeting remains to be studied.

In this issue many of the scientists who have been instrumental in elucidating the importance of epigenetics in pediatric brain tumor biology review the landscape of epigenetics across the spectrum of pediatric brain tumors. Over the next decade the pediatric neuro-oncology community is tasked with translating these important breakthroughs in the understanding of the unique biology of pediatric brain tumors into effective therapies for children with both aggressive brain tumors as well as more slowly growing tumors, which can still cause significant morbidity and even in selected situations, mortality.

\section{References}

1. Howlader NNA, Krapcho M, Miller D et al (2020) SEER cancer statistics review, 1975-2017. National Cancer Institute, Bethesda

2. Jones PA, Baylin SB (2002) The fundamental role of epigenetic events in cancer. Nat Rev Genet 3:415-428. https://doi. org/10.1038/nrg816

3. La Madrid AM, Kieran MW (2018) Epigenetics in clinical management of children and adolescents with brain tumors. Curr Cancer Drug Targets 18:57-64. https://doi.org/10.2174/1568009617 666170203164456

4. Jones C, Karajannis MA, Jones DTW et al (2017) Pediatric highgrade glioma: biologically and clinically in need of new thinking. Neuro Oncol 19:153-161. https://doi.org/10.1093/neuonc/now10 1

5. Jones DTW, Kieran MW, Bouffet E et al (2018) Pediatric lowgrade gliomas: next biologically driven steps. Neuro Oncol 20:160-173. https://doi.org/10.1093/neuonc/nox 141

6. Louis DN, Perry A, Reifenberger G et al (2016) The 2016 World Health Organization classification of tumors of the central nervous system: a summary. Acta Neuropathol 131:803-820. https://doi. org/10.1007/s00401-016-1545-1

7. Biegel JA, Kalpana G, Knudsen ES et al (2002) The role of INI1 and the SWI/SNF complex in the development of rhabdoid tumors: meeting summary from the workshop on childhood atypical teratoid/rhabdoid tumors. Cancer Res 62:323-328

8. Kohashi K, Oda Y (2017) Oncogenic roles of SMARCB1/INI1 and its deficient tumors. Cancer Sci 108:547-552. https://doi. org/10.1111/cas.13173

9. Wilson BG, Wang X, Shen X et al (2010) Epigenetic antagonism between polycomb and SWI/SNF complexes during oncogenic transformation. Cancer Cell 18:316-328. https://doi. org/10.1016/j.ccr.2010.09.006

10. Kurmasheva RT, Sammons M, Favours E et al (2017) Initial testing (stage 1) of tazemetostat (EPZ-6438), a novel EZH2 inhibitor, by the pediatric preclinical testing program. Pediatr Blood Cancer. https://doi.org/10.1002/pbc.26218

11. Chi S, Fouladi M, Shukla N et al (2018) Abstract A175: Phase 1 study of the EZH2 inhibitor, tazemetostat, in children with relapsed or refractory INI1-negative tumors including rhabdoid tumors, epithelioid sarcoma, chordoma, and synovial sarcoma. Mol Cancer Ther 17:A175-A175. https://doi.org/10.1158/15357163.targ-17-a175

12. Roussel MF, Stripay JL (2018) Epigenetic drivers in pediatric medulloblastoma. Cerebellum 17:28-36. https://doi.org/10.1007/ s12311-017-0899-9

13. Schwartzentruber J, Korshunov A, Liu XY et al (2012) Driver mutations in histone $\mathrm{H} 3.3$ and chromatin remodelling genes in paediatric glioblastoma. Nature 482:226-231. https://doi. org/10.1038/nature 10833

14. Khuong-Quang DA, Buczkowicz P, Rakopoulos P et al (2012) K27M mutation in histone H3.3 defines clinically and biologically distinct subgroups of pediatric diffuse intrinsic pontine gliomas. Acta Neuropathol 124:439-447. https://doi.org/10.1007/s0040 1-012-0998-0

15. Grasso CS, Tang Y, Truffaux N et al (2015) Functionally defined therapeutic targets in diffuse intrinsic pontine glioma. Nat Med 21:555-559. https://doi.org/10.1038/nm.3855

16. Hubner JM, Muller T, Papageorgiou DN et al (2019) EZHIP/ CXorf67 mimics K27M mutated oncohistones and functions as an intrinsic inhibitor of PRC2 function in aggressive posterior fossa ependymoma. Neuro Oncol 21:878-889. https://doi.org/10.1093/ neuonc/noz058 
17. Mack SC, Witt H, Piro RM et al (2014) Epigenomic alterations define lethal CIMP-positive ependymomas of infancy. Nature 506:445-450. https://doi.org/10.1038/nature13108

18. Bayliss J, Mukherjee P, Lu C et al (2016) Lowered H3K27me3 and DNA hypomethylation define poorly prognostic pediatric posterior fossa ependymomas. Sci Transl Med 8:366ra161. https ://doi.org/10.1126/scitranslmed.aah6904

19. Jain SU, Do TJ, Lund PJ et al (2019) PFA ependymoma-associated protein EZHIP inhibits PRC2 activity through a H3 K27M-like mechanism. Nat Commun 10:2146. https://doi.org/10.1038/s4146 7-019-09981-6

Publisher's Note Springer Nature remains neutral with regard to jurisdictional claims in published maps and institutional affiliations. 